\title{
Applications of double lacunary sequences to n-norm
}

\author{
Kuldip Raj \\ School of Mathematics \\ Shri Mata Vaishno Devi University \\ Katra-182320, J\&K, India \\ email: kuldipraj68@gmail.com
}

\author{
Sunil Kumar Sharma \\ School of Mathematics \\ Shri Mata Vaishno Devi University \\ Katra-182320, J\&K, India \\ email: sunilksharma42@yahoo.co.in
}

\begin{abstract}
In the present paper we define some classes of double lacunary sequence spaces over $n$-normed spaces by means of a Musielak- Orlicz function. We study some relevant algebraic and topological properties. Further some inclusion relation among the classes are also examined.
\end{abstract}

\section{Introduction and preliminaries}

The initial work on double sequences is found in Bromwich [4]. Out of the definitions of convergence commonly employed for double series, only that due to Pringsheim permits a series to converge conditionally. Therefore, in spite of any disadvantages which it may possess, this definition is better adapted than others for the study of many problems in double sequences and series. Chief among the reasons why the theory of double sequences, under the Pringsheim definition of convergence, presents difficulties not encountered in the theory of simple sequences is the fact that a double sequence $\left\{x_{i j}\right\}$ may converge without $x_{i j}$ being a bounded function of $i$ and $j$. Thus it is not surprising that many authors in dealing with the convergence of double sequences should have restricted themselves to the class of bounded sequences, or in dealing with the

2010 Mathematics Subject Classification: 40A05, 40C05, 46A45

Key words and phrases: P-convergent, lacunary sequence, Orlicz function, sequence spaces, paranorm space, $n$-normed space 
summability of double series, to the class of series for which the function whose limit is the sum of the series is a bounded function of $i$ and $j$. Without such a restriction, peculiar things may sometimes happen; for example, a double power series may converge with partial sum $\left\{S_{i j}\right\}$ unbounded at a place exterior to its associated circles of convergence. Nevertheless there are problems in the theory of double sequences and series where this restriction of boundedness as it has been applied is considerably more stringent than need be. After Bromwich, the study of double sequences was initiated by Hardy [11], Moricz [26], Moricz and Rhoades [19], Tripathy ([35], [36]), Basarir and Sonalcan [2] and many others. Hardy [11] introduced the notion of regular convergence for double sequences. Quite recently, Zeltser [38] in her Ph.D thesis has essentially studied both the theory of topological double sequence spaces and the theory of summability of double sequences.

In order to extend the notion of convergence of sequences, statistical convergence was introduced by Schoenberg [34] and the idea depends on the notion of density [31] of subset of $\mathbb{N}$. Mursaleen and Edely [23] have recently introduced the statistical convergence and Cauchy convergence for double sequences and given the relation between statistical convergent and strongly Cesaro summable double sequences. Nextly, Mursaleen [21] and Mursaleen and Edely [24] have defined the almost strong regularity of matrices for double sequences and applied these matrices to establish a core theorem and introduced the $\mathrm{M}$-core for double sequences and determined those four dimensional matrices transforming every bounded double sequences $x=\left(x_{m n}\right)$ into one whose core is a subset of the $M$-core of $x$. More recently, Altay and Basar [1] have defined the spaces $\mathcal{B S}, \mathcal{B S}(\mathrm{t}), \mathcal{C} \mathcal{S}_{\mathrm{p}}, \mathcal{C} \mathcal{S}_{\mathrm{bp}}, \mathcal{C} \mathcal{S}_{\mathrm{r}}$ and $\mathcal{B V}$ of double sequences consisting of all double series whose sequence of partial sums are in the spaces $\mathcal{M}_{\mathfrak{u}}$, $\mathcal{M}_{\mathfrak{u}}(\mathrm{t}), \mathcal{C}_{\mathrm{p}}, \mathcal{C}_{\mathrm{bp}}, \mathcal{C}_{\mathrm{r}}$ and $\mathcal{L}_{\mathfrak{u}}$, respectively and also examined some properties of these sequence spaces and determined the $\alpha$-duals of the spaces $\mathcal{B S}, \mathcal{B V}, \mathcal{C} \mathcal{S}_{\mathrm{bp}}$ and the $\beta(v)$-duals of the spaces $\mathcal{C} \mathcal{S}_{\mathrm{bp}}$ and $\mathcal{C} \mathcal{S}_{\mathrm{r}}$ of double series. Now, recently Basar and Sever [3] have introduced the Banach space $\mathcal{L}_{\mathrm{q}}$ of double sequences corresponding to the well known space $\ell_{\mathrm{q}}$ of single sequences and examined some properties of the space $\mathcal{L}_{\mathrm{q}}$. By the convergence of a double sequence we mean the convergence in the Pringsheim sense i.e. a double sequence $x=\left(x_{k l}\right)$ has Pringsheim limit $\mathrm{L}$ (denoted by $\mathrm{P}-\lim x=\mathrm{L}$ ) provided that given $\epsilon>0$ there exists $n \in N$ such that $\left|x_{k l}-L\right|<\epsilon$ whenever $k, l>n$ see [27]. We shall write more briefly as $\mathrm{P}$-convergent. The double sequence $\mathrm{x}=\left(\mathrm{x}_{\mathrm{kl}}\right)$ is bounded if there exists a positive number $M$ such that $\left|x_{k l}\right|<M$ for all $k$ and $l$. The notion of difference sequence spaces was introduced by Kizmaz [12], who studied the difference sequence spaces $l_{\infty}(\Delta), c(\Delta)$ and $c_{0}(\Delta)$. The notion was 
further generalized by Et. and Colak [5] by introducing the spaces $l_{\infty}\left(\Delta^{\mathfrak{n}}\right)$, $c\left(\Delta^{n}\right)$ and $c_{0}\left(\Delta^{n}\right)$. Let $w$ be the space of all complex or real sequences $x=\left(x_{k}\right)$ and let $m, s$ be non-negative integers, then for $Z=l_{\infty}, c, c_{0}$ we have sequence spaces

$$
\mathrm{Z}\left(\Delta^{\mathrm{m}}\right)=\left\{x=\left(x_{\mathrm{k}}\right) \in w:\left(\Delta^{\mathrm{m}} \mathrm{x}_{\mathrm{k}}\right) \in \mathrm{Z}\right\},
$$

where $\Delta^{m} x=\left(\Delta^{m} x_{k}\right)=\left(\Delta^{m-1} x_{k}-\Delta^{m-1} x_{k+1}\right)$ and $\Delta^{0} x_{k}=x_{k}$ for all $k \in \mathbb{N}$, which is equivalent to the following binomial representation

$$
\Delta^{\mathrm{m}} \mathrm{x}_{\mathrm{k}}=\sum_{\nu=0}^{\mathrm{m}}(-1)^{v}\left(\begin{array}{c}
\mathrm{m} \\
v
\end{array}\right) \mathrm{x}_{\mathrm{k}+v} .
$$

Taking $m=1$, we get the spaces which were introduced and studied by Kizmaz [12].

An orlicz function $M:[0, \infty) \rightarrow[0, \infty)$ is a continuous, non-decreasing and convex function such that $M(0)=0, M(x)>0$ for $x>0$ and $M(x) \longrightarrow \infty$ as $x \longrightarrow \infty$.

Lindenstrauss and Tzafriri [14] used the idea of Orlicz function to define the following sequence space,

$$
\ell_{M}=\left\{x \in w: \sum_{k=1}^{\infty} M\left(\frac{\left|x_{k}\right|}{\rho}\right)<\infty, \text { for some } \rho>0\right\}
$$

which is called as an Orlicz sequence space. Also $\ell_{M}$ is a Banach space with the norm

$$
\|x\|=\inf \left\{\rho>0: \sum_{k=1}^{\infty} M\left(\frac{\left|x_{k}\right|}{\rho}\right) \leq 1\right\} .
$$

Also, it was shown in [14] that every Orlicz sequence space $\ell_{M}$ contains a subspace isomorphic to $\ell_{p}(p \geq 1)$. The $\Delta_{2-}$ condition is equivalent to $M(L x) \leq$ $\mathrm{LM}(\mathrm{x})$, for all $\mathrm{L}$ with $0<\mathrm{L}<1$. An Orlicz function $M$ can always be represented in the following integral form

$$
M(x)=\int_{0}^{x} \eta(t) d t
$$

where $\eta$ is known as the kernel of $M$, is right differentiable for $t \geq 0, \eta(0)=$ $0, \eta(t)>0, \eta$ is non-decreasing and $\eta(t) \rightarrow \infty$ as $t \rightarrow \infty$.

Let $X$ be a linear metric space. A function $p: X \rightarrow \mathbb{R}$ is called paranorm, if

1. $p(x) \geq 0$ for all $x \in X$, 
2. $p(-x)=p(x)$ for all $x \in X$,

3. $p(x+y) \leq p(x)+p(y)$ for all $x, y \in X$,

4. if $\left(\lambda_{n}\right)$ is a sequence of scalars with $\lambda_{n} \rightarrow \lambda$ as $n \rightarrow \infty$ and $\left(x_{n}\right)$ is a sequence of vectors with $p\left(x_{n}-x\right) \rightarrow 0$ as $n \rightarrow \infty$, then $p\left(\lambda_{n} x_{n}-\lambda x\right) \rightarrow$ 0 as $n \rightarrow \infty$.

A paranorm $p$ for which $p(x)=0$ implies $x=0$ is called total paranorm and the pair $(X, p)$ is called a total paranormed space. It is well known that the metric of any linear metric space is given by some total paranorm (see [37], Theorem 10.4.2, pp. 183). For more details about sequence spaces see ([17], [22], [25], [28], [29], [30], [32], [33]) and reference therein.

Let $\ell_{\infty}, c$ and $c_{0}$ denotes the sequence spaces of bounded, convergent and null sequences $x=\left(x_{k}\right)$ respectively. A sequence $x=\left(x_{k}\right) \in \ell_{\infty}$ is said to be almost convergent if all Banach limits of $x=\left(x_{k}\right)$ coincide. In [13], it was shown that

$$
\hat{c}=\left\{x=\left(x_{k}\right): \lim _{n \rightarrow \infty} \frac{1}{n} \sum_{k=1}^{n} x_{k+s} \text { exists, uniformly in } s\right\} \text {. }
$$

In ([15], [16]) Maddox defined strongly almost convergent sequences. Recall that a sequence $x=\left(x_{k}\right)$ is strongly almost convergent if there is a number $L$ such that

$$
\lim _{n \rightarrow \infty} \frac{1}{n} \sum_{k=1}^{n}\left|x_{k+s}-L\right|=0 \text {, uniformly in } s .
$$

By a lacunary sequence $\theta=\left(i_{r}\right), r=0,1,2, \cdots$, where $i_{0}=0$, we shall mean an increasing sequence of non-negative integers $g_{r}=\left(i_{r}-i_{r-1}\right) \rightarrow \infty \quad(r \rightarrow$ $\infty)$. The intervals determined by $\theta$ are denoted by $I_{r}=\left(i_{r-1}, i_{r}\right]$ and the ratio $i_{r} / i_{r-1}$ will be denoted by $n_{r}$. The space of lacunary strongly convergent sequences $N_{\theta}$ was defined by Freedman [6] as follows:

$$
\mathrm{N}_{\theta}=\left\{x=\left(x_{k}\right): \lim _{\mathrm{r} \rightarrow \infty} \frac{1}{\mathrm{~g}_{\mathrm{r}}} \sum_{\mathrm{k} \in \mathrm{I}_{\mathrm{r}}}\left|x_{\mathrm{k}}-\mathrm{L}\right|=0 \text { for some } \mathrm{L}\right\} .
$$

The double sequence $\theta_{r, s}=\left\{\left(k_{r}, l_{s}\right)\right\}$ is called double lacunary if there exist two increasing sequences of integers such that

$$
k_{0}=0, g_{r}=k_{r}-k_{r-1} \rightarrow \infty \text { as } r \rightarrow \infty
$$

and

$$
l_{0}=0, \bar{g}_{s}=l_{s}-l_{s-1} \rightarrow \infty \text { as } s \rightarrow \infty .
$$


Let $k_{r, s}=k_{r} l_{s}, g_{r, s}=g_{r} \bar{g}_{s}$ and $\theta_{r, s}$ is determined by $I_{r, s}=\left\{(k, l): k_{r-1}<k \leq\right.$ $\left.k_{\mathrm{r}} \& \mathrm{l}_{\mathrm{s}-1}<\mathrm{l} \leq \mathrm{l}_{\mathrm{s}}\right\}, \mathrm{q}_{\mathrm{r}}=\frac{\mathrm{k}_{\mathrm{r}}}{\mathrm{k}_{\mathrm{r}-1}}, \overline{\mathrm{q}}_{\mathrm{s}}=\frac{\mathrm{l}_{\mathrm{s}}}{l_{\mathrm{s}-1}}$ and $\mathrm{q}_{\mathrm{r}, \mathrm{s}}=\mathrm{q}_{\mathrm{r}} \overline{\mathrm{q}}_{\mathrm{s}}$.

The concept of 2-normed spaces was initially developed by Gähler [7] in the mid of 1960's, while that of n-normed spaces one can see in Misiak [20]. Since then, many others have studied this concept and obtained various results, see Gunawan ([8],[9]) and Gunawan and Mashadi [10]. Let $\boldsymbol{n} \in \mathbb{N}$ and $X$ be a linear space over the field $\mathbb{R}$ of reals of dimension $d$, where $d \geq n \geq 2$. A real valued function $\|\cdot, \cdots, \cdot\|$ on $X^{n}$ satisfying the following four conditions:

1. $\left\|x_{1}, x_{2}, \cdots, x_{n}\right\|=0$ if and only if $x_{1}, x_{2}, \cdots, x_{n}$ are linearly dependent in $X$;

2. $\left\|x_{1}, x_{2}, \cdots, x_{n}\right\|$ is invariant under permutation;

3. $\left\|\alpha x_{1}, x_{2}, \cdots, x_{n}\right\|=|\alpha|\left\|x_{1}, x_{2}, \cdots, x_{n}\right\|$ for any $\alpha \in \mathbb{R}$, and

4. $\left\|x+x^{\prime}, x_{2}, \cdots, x_{n}\right\| \leq\left\|x, x_{2}, \cdots, x_{n}\right\|+\left\|x^{\prime}, x_{2}, \cdots, x_{n}\right\|$

is called a $n$-norm on $X$, and the pair $(X,\|\cdot, \cdots, \cdot\|)$ is called a $n$-normed space over the field $\mathbb{R}$.

For example, we may take $X=\mathbb{R}^{n}$ being equipped with the Euclidean $n$-norm $\left\|x_{1}, x_{2}, \cdots, x_{n}\right\|_{E}=$ the volume of the $n$-dimensional parallelopiped spanned by the vectors $x_{1}, x_{2}, \cdots, x_{n}$ which may be given explicitly by the formula

$$
\left\|x_{1}, x_{2}, \cdots, x_{n}\right\|_{E}=\left|\operatorname{det}\left(x_{i j}\right)\right| \text {, }
$$

where $x_{i}=\left(x_{i 1}, x_{i 2}, \cdots, x_{i n}\right) \in \mathbb{R}^{n}$ for each $i=1,2, \cdots, n$. Let $(X,\|, \cdots, \cdot\|)$ be a $n$-normed space of dimension $d \geq n \geq 2$ and $\left\{a_{1}, a_{2}, \cdots, a_{n}\right\}$ be linearly independent set in $X$. Then the following function $\|\cdot, \cdots, \cdot\|_{\infty}$ on $X^{n-1}$ defined by

$$
\left\|x_{1}, x_{2}, \cdots, x_{n-1}\right\|_{\infty}=\max \left\{\left\|x_{1}, x_{2}, \cdots, x_{n-1}, a_{i}\right\|: i=1,2, \cdots, n\right\}
$$

defines an $(n-1)$-norm on $X$ with respect to $\left\{a_{1}, a_{2}, \cdots, a_{n}\right\}$. A sequence $\left(x_{k}\right)$ in a $n$-normed space $(X,\|\cdot, \cdots, \cdot\|)$ is said to converge to some $\mathrm{L} \in \mathrm{X}$ if

$$
\lim _{k \rightarrow \infty}\left\|x_{k}-L, z_{1}, \cdots, z_{n-1}\right\|=0 \text { for every } z_{1}, \cdots, z_{n-1} \in X .
$$

A sequence $\left(x_{k}\right)$ in a $n$-normed space $(X,\|\cdot, \cdots, \cdot\|)$ is said to be Cauchy if

$$
\lim _{k, p \rightarrow \infty}\left\|x_{k}-x_{p}, z_{1}, \cdots, z_{n-1}\right\|=0 \text { for every } z_{1}, \cdots, z_{n-1} \in X .
$$


If every Cauchy sequence in $X$ converges to some $L \in X$, then $X$ is said to be complete with respect to the $n$-norm. Any complete $n$-normed space is said to be $n$-Banach space.

Let $(X,\|\cdot, \cdots, \cdot\|)$ be a real $n$-normed space and $w(n-X)$ denotes the space of $X$-valued sequences. Let $p=\left(p_{k, l}\right)$ be any bounded sequence of positive real numbers, $d=\left(d_{k, l}\right)$ be any sequence of strictly positive real numbers and $\mathcal{M}=\left(M_{k, l}\right)$ be a sequence of Orlicz functions. In this paper we define the following sequence spaces:

$$
\begin{aligned}
& {\left[c^{2}, \mathcal{M}, p, d,\|\cdot, \cdots, \cdot\|\right]^{\theta}\left(\Delta^{m}\right)=\left\{x=\left(x_{k, l}\right) \in w(n-X):\right.} \\
& \lim _{r, s \rightarrow \infty} \frac{1}{g_{r, s}} \sum_{k, l \in I_{r, s}}\left[M_{k, l}\left(\left\|\frac{d_{k, l} \Delta^{m} x_{k+u, l+v}-L}{\rho}, z_{1}, \cdots, z_{n-1}\right\|\right)\right]^{p_{k, l}}=0, \\
& \text { uniformly in u and } \left.v, z_{1}, \cdots, z_{n-1} \in X, \text { for some L and } \rho>0\right\}, \\
& {\left[c^{2}, \mathcal{M}, p, d,\|\cdot, \cdots, \cdot\|\right]_{0}^{\theta}\left(\Delta^{m}\right)=\left\{x=\left(x_{k, l}\right) \in \mathcal{w}(n-X):\right.} \\
& \lim _{r, s \rightarrow \infty} \frac{1}{g_{r, s}} \sum_{k, l \in I_{r, s}}\left[M_{k, l}\left(\left\|\frac{d_{k, l} \Delta^{m} x_{k+u, l+v}}{\rho}, z_{1}, \cdots, z_{n-1}\right\|\right)\right]^{p_{k, l}}=0, \\
& \left.\quad u_{i n i f o r m l y} \text { in } u \text { and } v, z_{1}, \cdots, z_{n-1} \in X \text { and } \rho>0\right\} \text { and } \\
& {\left[c^{2}, \mathcal{M}, p, d,\|\cdot, \cdots, \cdot\|\right]_{\infty}^{\theta}\left(\Delta^{m}\right)=\left\{x=\left(x_{k, l}\right) \in w(n-X):\right.} \\
& \sup _{r, s} \frac{1}{g_{r, s}} \sum_{k, l \in I_{r, s}}\left[M_{k, l}\left(\left\|\frac{d_{k, l} \Delta^{m} x_{k+u, l+v}}{\rho}, z_{1}, \cdots, z_{n-1}\right\|\right)\right]^{p_{k, l}}<\infty,
\end{aligned}
$$

uniformly in $u$ and $v, z_{1}, \cdots, z_{n-1} \in X$ and $\left.\rho>0\right\}$.

When $\mathcal{M}(x)=x$, we get

$$
\begin{aligned}
& {\left[c^{2}, p, d,\|\cdot, \cdots, \cdot\|\right]^{\theta}\left(\Delta^{m}\right)=\left\{x=\left(x_{k, l}\right) \in w(n-X):\right.} \\
& \quad \lim _{r, s \rightarrow \infty} \frac{1}{g_{r, s}} \sum_{k, l \in I_{r, s}}\left(\left\|\frac{d_{k, l} \Delta^{m} x_{k+u, l+v}-L}{\rho}, z_{1}, \cdots, z_{n-1}\right\|\right)^{p_{k, l}}=0,
\end{aligned}
$$

uniformly in $u$ and $v, z_{1}, \cdots, z_{n-1} \in X$ for some $L$ and $\left.\rho>0\right\}$, $\left[\mathrm{c}^{2}, \mathrm{p}, \mathrm{d},\|\cdot, \cdots, \cdot\|\right]_{0}^{\theta}\left(\Delta^{\mathrm{m}}\right)=\left\{\mathrm{x}=\left(\mathrm{x}_{\mathrm{k}, \mathrm{l}}\right) \in \mathcal{w}(\mathrm{n}-\mathrm{X})\right.$ :

$$
\lim _{r, s \rightarrow \infty} \frac{1}{g_{r, s}} \sum_{k, l \in I_{r, s}}\left(\left\|\frac{d_{k, l} \Delta^{m} x_{k+u, l+v}}{\rho}, z_{1}, \cdots, z_{n-1}\right\|\right)^{p_{k, l}}=0,
$$

uniformly in $u$ and $v, z_{1}, \cdots, z_{\mathfrak{n}-1} \in X$ and $\left.\rho>0\right\}$ 
and

$$
\begin{aligned}
& {\left[c^{2}, p, d,\|\cdot, \cdots, \cdot\|\right]_{\infty}^{\theta}\left(\Delta^{m}\right)=\left\{x=\left(x_{k, l}\right) \in w(n-X):\right.} \\
& \quad \sup _{r, s} \frac{1}{g_{r, s}} \sum_{k, l \in I_{r, s}}\left(\left\|\frac{d_{k, l} \Delta^{m} x_{k+u, l+v}}{\rho}, z_{1}, \cdots, z_{n-1}\right\|\right)^{p_{k, l}}<\infty
\end{aligned}
$$

uniformly in $u$ and $v, z_{1}, \cdots, z_{n-1} \in X$ and $\left.\rho>0\right\}$.

If we take $p=\left(p_{k, l}\right)=1$ and $d=\left(d_{k, l}\right)=1$ for all $k, l$ then we get

$$
\begin{aligned}
& {\left[c^{2}, \mathcal{M},\|\cdot, \cdots, \cdot\|\right]^{\theta}\left(\Delta^{m}\right)=\left\{x=\left(x_{k, l}\right) \in w(n-X):\right.} \\
& \quad \lim _{r, s \rightarrow \infty} \frac{1}{g_{r, s}} \sum_{k, l \in I_{r, s}}\left[M_{k, l}\left(\left\|\frac{\Delta^{m} x_{k+u, l+v}-L}{\rho}, z_{1}, \cdots, z_{n-1}\right\|\right)\right]=0,
\end{aligned}
$$

uniformly in $u$ and $v, z_{1}, \cdots, z_{n-1} \in X$, for some $L$ and $\left.\rho>0\right\}$,

$$
\begin{aligned}
& {\left[c^{2}, \mathcal{M},\|\cdot, \cdots, \cdot\|\right]_{0}^{\theta}\left(\Delta^{m}\right)=\left\{x=\left(x_{k, l}\right) \in w(n-X):\right.} \\
& \quad \lim _{r, s \rightarrow \infty} \frac{1}{g_{r, s}} \sum_{k, l \in I_{r}, s}\left[M_{k, l}\left(\left\|\frac{\Delta^{m} x_{k+u, l+v}}{\rho}, z_{1}, \cdots, z_{n-1}\right\|\right)\right]=0
\end{aligned}
$$

uniformly in $u$ and $v, z_{1}, \cdots, z_{\mathfrak{n}-1} \in X$ and $\left.\rho>0\right\}$ and

$$
\begin{aligned}
& {\left[c^{2}, \mathcal{M},\|\cdot, \cdots, \cdot\|\right]_{\infty}^{\theta}\left(\Delta^{m}\right)=\left\{x=\left(x_{k, l}\right) \in w(n-X):\right.} \\
& \sup _{r, s} \frac{1}{g_{r, s}} \sum_{k, l \in I_{r, s}}\left[M_{k, l}\left(\left\|\frac{\Delta^{m} x_{k+u, l+v}}{\rho}, z_{1}, \cdots, z_{n-1}\right\|\right)\right]<\infty,
\end{aligned}
$$

uniformly in $u$ and $v, z_{1}, \cdots, z_{\mathfrak{n}-1} \in X$ and $\left.\rho>0\right\}$.

The following inequality will be used throughout the paper. Let $p=\left(p_{k, l}\right)$ be a double sequence of positive real numbers with $0<p_{k, l} \leq \sup _{k, l} p_{k, l}=H$ and let $K=\max \left\{1,2^{\mathrm{H}-1}\right\}$. Then for the factorable sequences $\left\{a_{k, l}\right\}$ and $\left\{b_{k, l}\right\}$ in the complex plane, we have

$$
\left|a_{k, l}+b_{k, l}\right|^{p_{k, l}} \leq K\left(\left|a_{k, l}\right|^{p_{k, l}}+\left|b_{k, l}\right|^{p_{k, l}}\right)
$$

The aim of this paper is to introduce some new type of lacunary double sequence spaces defined by a sequence of Orlicz function $\mathcal{M}=\left(M_{k, l}\right)$ over $n$ normed spaces and to establish some topological properties and some inclusion relation between above defined sequence spaces. 


\section{Main results}

Theorem 1 Let $\mathcal{M}=\left(M_{k, l}\right)$ be a sequence of Orlicz functions, $\mathrm{p}=\left(\mathrm{p}_{\mathrm{k}, \mathrm{l}}\right)$ be a bounded sequence of positive real numbers and $\mathrm{d}=\left(\mathrm{d}_{\mathrm{k}, \mathrm{l}}\right)$ be a sequence of strictly positive real numbers. Then the sequence spaces $\left[\mathrm{c}^{2}, \mathcal{M}, \mathrm{p}, \mathrm{d},\|\cdot, \cdots, \cdot\|\right]^{\Theta}$ $\left(\Delta^{\mathrm{m}}\right),\left[\mathrm{c}^{2}, \mathcal{M}, \mathrm{p}, \mathrm{d},\|\cdot, \cdots, \cdot\|\right]_{0}^{\theta}\left(\Delta^{\mathrm{m}}\right)$ and $\left[\mathrm{c}^{2}, \mathcal{M}, \mathrm{p}, \mathrm{d},\|\cdot, \cdots, \cdot\|\right]_{\infty}^{\theta}\left(\Delta^{\mathrm{m}}\right)$ are linear spaces over the field of real numbers $\mathbb{R}$.

Proof. Let $x=\left(x_{k, l}\right), y=\left(y_{k, l}\right) \in\left[c^{2}, \mathcal{M}, p, d,\|\cdot, \cdots, \cdot\|\right]_{0}^{\theta}\left(\Delta^{m}\right)$ and $\alpha, \beta \in \mathbb{R}$. Then there exist positive numbers $\rho_{1}$ and $\rho_{2}$ such that

$$
\lim _{r, s \rightarrow \infty} \frac{1}{g_{r, s}} \sum_{k, l \in I_{r, s}}\left[M_{k, l}\left(\left\|\frac{d_{k, l} \Delta^{m} x_{k+u, l+v}}{\rho_{1}}, z_{1}, \cdots, z_{n-1}\right\|\right)\right]^{p_{k, l}}=0,
$$

uniformly in $u$ and $v$, and

$$
\lim _{r, s \rightarrow \infty} \frac{1}{g_{r, s}} \sum_{k, l \in I_{r, s}}\left[M_{k, l}\left(\left\|\frac{d_{k, l} \Delta^{m} y_{k+u, l+v}}{\rho_{2}}, z_{1}, \cdots, z_{n-1}\right\|\right)\right]^{p_{k, l}}=0,
$$

uniformly in $u$ and $v$.

Let $\rho_{3}=\max \left(2|\alpha| \rho_{1}, 2|\beta| \rho_{2}\right)$. Since $\mathcal{M}=\left(M_{k, l}\right)$ is non-decreasing and convex function so by using inequality (1), we have

$$
\begin{aligned}
& \frac{1}{g_{r, s}} \sum_{k, l \in I_{r}, s}\left[M_{k, l}\left(\left\|\frac{d_{k, l} \Delta^{m}\left(\alpha x_{k+u, l+v}+\beta y_{k+u, l+v}\right)}{\rho_{3}}, z_{1}, \cdots, z_{n-1}\right\|\right)\right]^{p_{k, l}} \\
& =\frac{1}{g_{r, s}} \sum_{k, l \in I_{r, s}}\left[M _ { k , l } \left(\left\|\frac{d_{k, l} \alpha \Delta^{m}\left(x_{k+u, l+v}\right)}{\rho_{3}}, z_{1}, \cdots, z_{n-1}\right\|\right.\right. \\
& \left.\left.+\left\|\frac{d_{k, l} \beta \Delta^{m}\left(y_{k+u, l+v}\right)}{\rho_{3}}, z_{1}, \cdots, z_{n-1}\right\|\right)\right]^{p_{k, l}} \\
& \leq K \frac{1}{g_{r, s}} \sum_{k, l \in I_{r, s}} \frac{1}{2^{p_{k, l}}}\left[M_{k, l}\left(\left\|\frac{d_{k, l} \Delta^{m}\left(x_{k+u, l+v}\right)}{\rho_{1}}, z_{1}, \cdots, z_{n-1}\right\|\right)\right]^{p_{k, l}} \\
& +K \frac{1}{g_{r, s}} \sum_{k, l \in I_{r, s}} \frac{1}{2_{k, l}}\left[M_{k, l}\left(\left\|\frac{d_{k, l} \Delta^{m}\left(y_{k+u, l+v}\right)}{\rho_{2}}, z_{1}, \cdots, z_{n-1}\right\|\right)\right]^{p_{k, l}} \\
& \leq \mathrm{K} \frac{1}{g_{r, s}} \sum_{k, l \in I_{r, s}}\left[M_{k, l}\left(\left\|\frac{d_{k, l} \Delta^{m}\left(x_{k+u, l+v}\right)}{\rho_{1}}, z_{1}, \cdots, z_{n-1}\right\|\right)\right]^{p_{k, l}} \\
& \quad+K \frac{1}{g_{r, s}} \sum_{k, l \in I_{r, s}}\left[M_{k, l}\left(\left\|\frac{d_{k, l} \Delta^{m}\left(y_{k+u, l+v}\right)}{\rho_{2}}, z_{1}, \cdots, z_{n-1}\right\|\right)\right]^{p_{k, l}}
\end{aligned}
$$


$\longrightarrow 0$ as $r \longrightarrow \infty, s \longrightarrow \infty$ uniformly in $u$ and $v$.

Thus, we have $\alpha x+\beta y \in\left[c^{2}, \mathcal{M}, p, d,\|\cdot, \cdots, \cdot\|_{0}^{\theta}\left(\Delta^{m}\right)\right.$. Hence $\left[c^{2}, \mathcal{M}, p, d\right.$, $\|\cdot, \cdots, \cdot\|]_{0}^{\theta}\left(\Delta^{\mathrm{m}}\right)$ is a linear space. Similarly, we can prove that $\left[\mathrm{c}^{2}, \mathcal{M}, \mathrm{p}, \mathrm{d}\right.$, $\|\cdot, \cdots, \cdot\|]^{\theta}\left(\Delta^{\mathrm{m}}\right)$ and $\left[\mathrm{c}^{2}, \mathcal{M}, \mathrm{p}, \mathrm{d},\|\cdot, \cdots, \cdot\|\right]_{\infty}^{\theta}\left(\Delta^{\mathrm{m}}\right)$ are linear spaces.

Theorem 2 Suppose $\mathcal{M}=\left(M_{k, l}\right)$ is a sequence of Orlicz functions, $p=\left(p_{k, l}\right)$ be a bounded sequence of positive real numbers and $\mathrm{d}=\left(\mathrm{d}_{\mathrm{k}, \mathrm{l}}\right)$ be a sequence of strictly positive real numbers, then $\left[\mathrm{c}^{2}, \mathcal{M}, \mathrm{p}, \mathrm{d},\|\cdot, \cdots, \cdot\|\right]_{0}^{\theta}\left(\Delta^{\mathrm{m}}\right)$ is a topological linear space paranormed by

$$
\begin{aligned}
g(x) & =\inf \left\{\rho^{\frac{p_{r, s}}{H}}:\left(\frac{1}{g_{r, s}} \sum_{k, l \in I_{r}, s}\left[M_{k, l}\left(\left\|\frac{d_{k, l} \Delta^{m} \chi_{k+u, l+v}}{\rho}, z_{1}, \cdots, z_{n-1}\right\|\right)\right]^{p_{k, l}}\right)^{\frac{1}{H}}\right. \\
& \leq 1, r, s \in \mathbb{N}\},
\end{aligned}
$$

where $\mathrm{H}=\max \left(1, \sup p_{k, l}\right)<\infty$.

Proof. Clearly $g(x) \geq 0$ for $x=\left(x_{k, l}\right) \in\left[c^{2}, \mathcal{M}, p, d,\|\cdot, \cdots, \cdot\|\right]_{0}^{\theta}\left(\Delta^{m}\right)$. Since $M_{k, l}(0)=0$, we get $g(0)=0$. Again, if $g(x)=0$, then

$$
\begin{aligned}
& \inf \left\{\rho^{\frac{p_{r, s}}{H}}:\left(\frac{1}{g_{r, s}} \sum_{k, l \in I_{r, s}}\left[M_{k, l}\left(\left\|\frac{d_{k, l} \Delta^{m} x_{k+u, l+v}}{\rho}, z_{1}, \cdots, z_{n-1}\right\|\right)\right]^{p_{k, l}}\right)^{\frac{1}{H}}\right. \\
& \leq 1, r, s \in \mathbb{N}\}=0 .
\end{aligned}
$$

This implies that for a given $\epsilon>0$, there exists some $\rho_{\epsilon}\left(0<\rho_{\epsilon}<\epsilon\right)$ such that

$$
\left(\frac{1}{g_{r, s}} \sum_{k, l \in I_{r, s}}\left[M_{k, l}\left(\left\|\frac{d_{k, l} \Delta^{m} x_{k+u, l+v}}{\rho_{\epsilon}}, z_{1}, \cdots, z_{n-1}\right\|\right)\right]^{p_{k, l}}\right)^{\frac{1}{H}} \leq 1 .
$$

Thus,

$$
\begin{aligned}
& \left(\frac{1}{g_{r, s}} \sum_{k, l \in I_{r, s}}\left[M_{k, l}\left(\left\|\frac{d_{k, l} \Delta^{m} x_{k+u, l+v}}{\epsilon}, z_{1}, \cdots, z_{n-1}\right\|\right)\right]^{p_{k, l}}\right)^{\frac{1}{H}} \\
& \leq\left(\frac{1}{g_{r, s}} \sum_{k, l \in I_{r, s}}\left[M_{k, l}\left(\left\|\frac{d_{k, l} \Delta^{m} x_{k+u, l+v}}{\rho_{\epsilon}}, z_{1}, \cdots, z_{n-1}\right\|\right)\right]^{p_{k, l}}\right)^{\frac{1}{H}} \leq 1,
\end{aligned}
$$


for each $r, s, u$ and $v$. Suppose that $x_{k, l} \neq 0$ for each $k, l \in \mathbb{N}$. This implies that $\mathrm{d}_{k, l} \Delta^{\mathrm{m}} \chi_{k+u, l+v} \neq 0$, for each $k, l, u, v \in \mathbb{N}$. Let $\epsilon \rightarrow 0$, then $\| \frac{d_{k, l} \Delta^{m} \chi_{k+u, l+v}}{\epsilon}, z_{1}, \cdots$, $z_{n-1} \| \rightarrow \infty$. It follows that

$$
\left(\frac{1}{g_{r, s}} \sum_{k, l \in I_{r, s}}\left[M_{k, l}\left(\left\|\frac{d_{k, l} \Delta^{m} \chi_{k+u, l+v}}{\epsilon}, z_{1}, \cdots, z_{n-1}\right\|\right)\right]^{p_{k, l}}\right)^{\frac{1}{H}} \rightarrow \infty
$$

which is a contradiction. Therefore, $d_{k, l} \Delta^{m} x_{k+u, l+v}=0$ for each $k, l, u$ and $v$ and thus $x_{k, l}=0$ for each $k, l \in \mathbb{N}$. Let $\rho_{1}>0$ and $\rho_{2}>0$ be such that

$$
\left(\frac{1}{g_{r, s}} \sum_{k, l \in I_{r, s}}\left[M_{k, l}\left(\left\|\frac{d_{k, l} \Delta^{m} x_{k+u, l+v}}{\rho_{1}}, z_{1}, \cdots, z_{n-1}\right\|\right)\right]^{p_{k, l}}\right)^{\frac{1}{H}} \leq 1
$$

and

$$
\left(\frac{1}{g_{r, s}} \sum_{k, l \in I_{r, s}}\left[M_{k, l}\left(\left\|\frac{d_{k, l} \Delta^{m} x_{k+u, l+v}}{\rho_{2}}, z_{1}, \cdots, z_{n-1}\right\|\right)\right]^{p_{k, l}}\right)^{\frac{1}{H}} \leq 1
$$

for each $r, s, u$ and $v$. Let $\rho=\rho_{1}+\rho_{2}$. Then, by Minkowski's inequality, we have

$$
\begin{aligned}
& \left(\frac{1}{g_{r, s}} \sum_{k, l \in I_{r, s}}\left[M_{k, l}\left(\left\|\frac{d_{k, l} \Delta^{m}\left(x_{k+u, l+v}+y_{k+u, l+v}\right)}{\rho}, z_{1}, \cdots, z_{n-1}\right\|\right)\right]^{p_{k, l}}\right)^{\frac{1}{H}} \\
& \leq\left(\frac{1}{g_{r, s}} \sum_{k, l \in I_{r, s}}\left[M_{k, l}\left(\left\|\frac{d_{k, l} \Delta^{m} x_{k+u, l+v}+d_{k, l} \Delta^{m} y_{k+u, l+v}}{\rho_{1}+\rho_{2}}, z_{1}, \cdots, z_{n-1}\right\|\right)\right]^{p_{k, l}}\right)^{\frac{1}{H}} \\
& \leq\left(\sum _ { k , l \in I _ { r , s } } \left[\frac{\rho_{1}}{\rho_{1}+\rho_{2}} M_{k, l}\left(\left\|\frac{d_{k, l} \Delta^{m} x_{k+u, l+v}}{\rho_{1}}, z_{1}, \cdots, z_{n-1}\right\|\right)\right.\right. \\
& \left.\left.\quad+\frac{\rho_{2}}{\rho_{1}+\rho_{2}} M_{k, l}\left(\left\|\frac{d_{k, l} \Delta^{m} y_{k+u, l+v}}{\rho_{2}}, z_{1}, \cdots, z_{n-1}\right\|\right)\right]^{p_{k, l}}\right) \frac{1}{H} \\
& \leq \\
& \left(\frac{\rho_{1}}{\rho_{1}+\rho_{2}}\right)\left(\frac{1}{g_{r, s}} \sum_{k, l \in I_{r, s}}\left[M_{k, l}\left(\left\|\frac{d_{k, l} \Delta^{m} x_{k+u, l+v}}{\rho_{1}}, z_{1}, \cdots, z_{n-1}\right\|\right)\right]^{p_{k, l}}\right)^{\frac{1}{H}} \\
& \quad+\left(\frac{\rho_{2}}{\rho_{1}+\rho_{2}}\right)\left(\frac{1}{g_{r, s}} \sum_{k, l \in I_{r, s}}\left[M_{k, l}\left(\left\|\frac{d_{k, l} \Delta^{m} y_{k+u, l+v}}{\rho_{2}}, z_{1}, \cdots, z_{n-1}\right\|\right)\right]^{p_{k, l}}\right)^{\frac{1}{H}} \\
& \leq 1 .
\end{aligned}
$$


Since $\rho^{\prime}$ s are non-negative, so we have

$$
\begin{aligned}
& g(x+y)=\inf \left\{\rho^{\frac{p_{r, s}}{H}}:\right. \\
& \left(\frac{1}{g_{r, s}} \sum_{k, l \in I_{r, s}}\left[M_{k, l}\left(\left\|\frac{d_{k, l} \Delta^{m}\left(x_{k+u, l+v}+y_{k+u, l+v}\right)}{\rho}, z_{1}, \cdots, z_{n-1}\right\|\right)\right]^{p_{k, l}}\right)^{\frac{1}{H}} \\
& \leq 1, \quad r, s, u, v \in \mathbb{N}\}, \\
& \leq \inf \left\{\rho_{1}^{\frac{p_{r, s}}{H}}:\left(\frac{1}{g_{r, s}} \sum_{k, l \in I_{r, s}}\left[M_{k, l}\left(\left\|\frac{d_{k, l} \Delta^{m} x_{k+u, l+v}}{\rho_{1}}, z_{1}, \cdots, z_{n-1}\right\|\right)\right]^{p_{k, l}}\right)^{\frac{1}{H}} \leq 1, r, s, u, v \in \mathbb{N}\right\} \\
& +\inf \left\{\rho_{2}^{\frac{p_{r, s}}{H}}:\left(\frac{1}{g_{r, s}} \sum_{k, l \in I_{r, s}}\left[M_{k, l}\left(\left\|\frac{d_{k, l} \Delta^{m^{m}} y_{k+u, l+v}}{\rho_{2}}, z_{1}, \cdots, z_{n-1}\right\|\right) g\right]^{p_{k, l}}\right)^{\frac{1}{H}}\right.
\end{aligned}
$$

Therefore,

$$
g(x+y) \leq g(x)+g(y) .
$$

Finally, we prove that the scalar multiplication is continuous. Let $\lambda$ be any complex number. By definition,

$$
\begin{gathered}
g(\lambda x)=\inf \left\{\rho^{\frac{p_{r, s}}{H}}:\left(\frac{1}{g_{r, s}} \sum_{k, l \in I_{r, s}}\left[M_{k, l}\left(\left\|\frac{d_{k, l} \Delta^{m} \lambda x_{k+u, l+v}}{\rho}, z_{1}, \cdots, z_{n-1}\right\|\right)\right]^{p_{k, l}}\right)^{\frac{1}{H}}\right. \\
\leq 1, r, s, u, v \in \mathbb{N}\} .
\end{gathered}
$$

Then

$$
\begin{aligned}
& g(\lambda x)=\inf \left\{(|\lambda| t)^{\frac{p_{r, s}}{H}}:\left(\frac{1}{g_{r, s}} \sum_{k, l \in I_{r, s}}\left[M_{k, l}\left(\left\|\frac{d_{k, l} \Delta^{m} \chi_{k+u, l+v}}{t}, z_{1}, \cdots, z_{n-1}\right\|\right)\right]^{p_{k, l}}\right)^{\frac{1}{H}}\right. \\
& \leq 1, r, s, u, v \in \mathbb{N}\},
\end{aligned}
$$

where $t=\frac{\rho}{|\lambda|}$. Since $|\lambda|^{\mathfrak{p}_{r, s}} \leq \max \left(1,|\lambda|^{\text {sup } p_{r, s}}\right)$, we have

$$
g(\lambda x) \leq \max \left(1,|\lambda|^{\text {sup }} \mathrm{p}_{\mathrm{r}, \mathrm{s}}\right)
$$




$$
\begin{aligned}
& \inf \left\{t^{\frac{p_{r, s}}{H}}:\left(\frac{1}{g_{r, s}} \sum_{k, l \in I_{r, s}}\left[M_{k, l}\left(\left\|\frac{d_{k, l} \Delta^{m} x_{k+u, l+v}}{t}, z_{1}, \cdots, z_{n-1}\right\|\right)\right]^{p_{k, l}}\right)^{\frac{1}{H}}\right. \\
& \leq 1, r, s, u, v \in \mathbb{N}\} .
\end{aligned}
$$

So, the fact that scalar multiplication is continuous follows from the above inequality. This completes the proof of the theorem.

Proposition 1 Let $\mathcal{M}=\left(M_{k, l}\right)$ be a sequence of Orlicz functions. If $\sup \left[M_{k, l}\right.$ $(\mathrm{x})]^{p_{k, l}}<\infty$ for all fixed $\mathrm{x}>0$, then $\left[\mathrm{c}^{2}, \mathcal{M}, \mathrm{p}, \mathrm{d},\|\cdot, \cdots, \cdot\|\right]_{0}^{\theta}\left(\Delta^{\mathrm{m}}\right) \subset\left[\mathrm{c}^{\mathrm{k}, \mathrm{l}}, \mathcal{M}, \mathrm{p}\right.$, $\mathrm{d},\|\cdot, \cdots, \cdot\|]_{\infty}^{\theta}\left(\Delta^{\mathrm{m}}\right)$.

Proof. Let $x=\left(x_{k, l}\right) \in\left[c^{2}, \mathcal{M}, p, d,\|\cdot, \cdots, \cdot\|\right]_{0}^{\theta}\left(\Delta_{m}\right)$, then there exists some positive $\rho_{1}$ such that

$$
\lim _{r, s \rightarrow \infty} \frac{1}{g_{r, s}} \sum_{k, l \in I_{r, s}}\left[M_{k, l}\left(\left\|\frac{d_{k, l} \Delta^{m} x_{k+u, l+v}}{\rho_{1}}, z_{1}, \cdots, z_{n-1}\right\|\right)\right]^{p_{k, l}}=0,
$$

uniformly in $u$ and $v$. Define $\rho=2 \rho_{1}$. Since $\mathcal{M}=\left(M_{k, l}\right)$ is non-decreasing and convex, by using inequality (1), we have

$$
\begin{aligned}
\sup _{r, s} & \frac{1}{g_{r, s}} \sum_{k, l \in I_{r, s}}\left[M_{k, l}\left(\left\|\frac{d_{k, l} \Delta^{m} \chi_{k+u, l+v}}{\rho}, z_{1}, \cdots, z_{n-1}\right\|\right)\right]^{p_{k, l}} \\
= & \sup _{r, s} \frac{1}{g_{r, s}} \sum_{k, l \in I_{r}, s}\left[M_{k, l}\left(\mid \frac{d_{k, l} \Delta^{m} x_{k+u, l+v}-L+L}{\rho}, z_{1}, \cdots, z_{n-1} \|\right)\right]^{p_{k, l}} \\
\leq & K \sup _{r, s} \frac{1}{g_{r, s}} \sum_{k, l \in I_{r, s}}\left[\frac{1}{2} M_{k, l}\left(\left\|\frac{d_{k, l} \Delta^{m} \chi_{k+u, l+v}-L}{\rho_{1}}, z_{1}, \cdots, z_{n-1}\right\|\right)\right]^{p_{k, l}} \\
& +K \sup _{r, s} \frac{1}{g_{r, s}} \sum_{k, l \in I_{r, s}}\left[\frac{1}{2} M_{k, l}\left(\| \frac{L}{\rho_{1}}, z_{1}, \cdots, z_{n-1} \mid\right)\right]^{p_{k, l}} \\
\leq & K \sup _{r, s} \frac{1}{g_{r, s}} \sum_{k, l \in I_{r, s}}\left[M_{k, l}\left(\mid \frac{d_{k, l} \Delta^{m} x_{k+u, l+v}-L}{\rho_{1}}, z_{1}, \cdots, z_{n-1} \|\right)\right]^{p_{k, l}} \\
& +K \sup _{r, s} \frac{1}{g_{r, s}} \sum_{k, l \in I_{r}, s}\left[M_{k, l}\left(\left\|\frac{L}{\rho_{1}}, z_{1}, \cdots, z_{n-1}\right\|\right)\right]^{p_{k, l}}<\infty .
\end{aligned}
$$

Hence $x=\left(x_{k, l}\right) \in\left[c^{2}, \mathcal{M}, p, d,\|\cdot, \cdots, \cdot\|\right]_{\infty}^{\theta}$. 
Theorem 3 Let $0<\inf p_{k, l}=h \leq p_{k, l} \leq \sup p_{k, l}=H<\infty$ and $\mathcal{M}=\left(M_{k, l}\right)$, $\mathcal{M}^{\prime}=\left(M_{\mathrm{k}, \mathrm{l}}^{\prime}\right)$ be two sequences of Orlicz functions satisfying $\Delta_{2}-$ condition, then we have

(i) $\left[c^{2}, \mathcal{M}^{\prime}, p, d,\|\cdot, \cdots, \cdot\|\right]_{0}^{\theta}\left(\Delta^{m}\right) \subset\left[c^{2}, \mathcal{M} \circ \mathcal{M}^{\prime}, p, d,\|\cdot, \cdots, \cdot\|\right]_{0}^{\theta}\left(\Delta^{m}\right)$,

(ii) $\left[c^{2}, \mathcal{M}^{\prime}, p, d,\|\cdot, \cdots, \cdot\|\right]^{\theta}\left(\Delta^{m}\right) \subset\left[c^{2}, \mathcal{M} \circ \mathcal{M}^{\prime}, p, d,\|\cdot, \cdots, \cdot\|\right]^{\theta}\left(\Delta^{m}\right)$ and

(iii) $\left[c^{2}, \mathcal{M}^{\prime}, p, d,\|\cdot, \cdots, \cdot\|\right]_{\infty}^{\theta}\left(\Delta^{\mathrm{m}}\right) \subset\left[c, \mathcal{M} \circ \mathcal{M}^{\prime}, p, d,\|\cdot, \cdots, \cdot\|\right]_{\infty}^{\theta}\left(\Delta^{\mathrm{m}}\right)$.

Proof. Let $x=\left(x_{k, l}\right) \in\left[c^{2}, \mathcal{M}^{\prime}, p, d,\|\cdot, \cdots, \cdot\|\right]_{0}^{\theta}\left(\Delta_{m}\right)$. Then we have

$$
\lim _{r, s \rightarrow \infty} \frac{1}{g_{r, s}} \sum_{k, l \in I_{r, s}}\left[M_{k, l}^{\prime}\left(\left\|\frac{d_{k, l} \Delta^{m} x_{k+u, l+v}}{\rho}, z_{1}, \cdots, z_{n-1}\right\|\right)\right]^{p_{k, l}}=0,
$$

uniformly in $u$ and $v$.

Let $\epsilon>0$ and choose $\delta$ with $0<\delta<1$ such that $M_{k, l}(t)<\epsilon$ for $0 \leq t \leq \delta$. Let

$$
y_{k+u, l+v}=M_{k, l}^{\prime}\left(\left\|\frac{d_{k, l} \Delta^{m} \chi_{k+u, l+v}}{\rho}, z_{1}, \cdots, z_{n-1}\right\|\right) \text { for all } k, l \in \mathbb{N} .
$$

We can write

$$
\begin{aligned}
\frac{1}{g_{r, s}} \sum_{k, l \in I_{r, s}}\left[M_{k, l}\left(y_{k+u, l+v}\right)\right]^{p_{k, l} l} & =\frac{1}{g_{r, s}} \sum_{\substack{k, l \in I_{r}, s \\
y_{k+u, l+v \leq \delta}}}\left[M_{k, l}\left(y_{k+u, l+v}\right)\right]^{p_{k, l}} \\
& +\frac{1}{g_{r, s}} \sum_{\substack{k, l \in I_{r}, s \\
y_{k+u, l+v \leq \delta}}}\left[M_{k, l}\left(y_{k+u, l+v}\right)\right]^{p_{k, l}} .
\end{aligned}
$$

Since $\mathcal{M}=\left(M_{k, l}\right)$ satisfying $\Delta_{2}$-condition, we have

$$
\begin{aligned}
& \frac{1}{g_{r, s}} \sum_{\substack{k, l \in I_{r}, s \\
y_{k+u, l+v \leq \delta}}}\left[M_{k, l}\left(y_{k+u, l+v}\right)\right]^{p_{k, l}} \\
& \leq\left[M_{k, l}(1)\right]^{H} \frac{1}{g_{r, s}} \sum_{\substack{k, l \in I_{r, s}, y_{k+u, l+v \leq \delta}}}\left[M_{k, l}\left(y_{k+u, l+v}\right)\right]^{p_{k, l}} \\
& \leq\left[M_{k, l}(2)\right]^{H} \frac{1}{g_{r, s}} \sum_{\substack{k, l \in I_{r}, s \\
y_{k+u, l+v \leq \delta}}}\left[M_{k, l}\left(y_{k+u, l+v}\right)\right]^{p_{k, l}} .
\end{aligned}
$$


For $y_{k+u, l+v}>\delta$, we have

$$
y_{k+u, l+v}<\frac{y_{k+u, l+v}}{\delta}<1+\frac{y_{k+u, l+v}}{\delta} .
$$

Since $\mathcal{M}=\left(M_{k, l}\right)$ is non-decreasing and convex, it follows that

$$
M_{k, l}\left(y_{k+u, l+v}\right)<M_{k, l}\left(1+\frac{y_{k+u, l+v}}{\delta}\right)<\frac{1}{2} M_{k, l}(2)+\frac{1}{2} M_{k, l}\left(\frac{2 y_{k+u, l+v}}{\delta}\right) .
$$

Since $\left(M_{k, l}\right)$ satisfies $\Delta_{2}$-condition, we can write

$$
\begin{aligned}
M_{k, l}\left(y_{k+u, l+v}\right) & <\frac{1}{2} T \frac{y_{k+u, l+v}}{\delta} M_{k, l}(2)+\frac{1}{2} T \frac{y_{k+u, l+v}}{\delta} M_{k, l}(2) \\
& =T \frac{y_{k+u, l+v}}{\delta} M_{k, l}(2) .
\end{aligned}
$$

Hence,

$$
\begin{aligned}
& \frac{1}{g_{r, s}} \sum_{\substack{k, l \in I_{r}, s \\
y_{k+u, l+v \leq \delta}}}\left[M_{k, l}\left(y_{k+u, l+v}\right)\right]^{p_{k, l}} \\
& \quad \leq \max \left(1,\left(\frac{\operatorname{TM}_{k, l}(2)}{\delta}\right)^{H}\right) \frac{1}{g_{r, s}} \sum_{\substack{k, l \in I_{r, s}, y_{k+u, l+v \leq \delta}}}\left[\left(y_{k+u, l+v}\right)\right]^{p_{k, l}}
\end{aligned}
$$

from equations (2) and (3), we have

$$
x=\left(x_{k, l}\right) \in\left[c^{2}, \mathcal{M} \circ \mathcal{M}^{\prime}, p, d,\|\cdot, \cdots, \cdot\|\right]_{0}^{\theta}\left(\Delta^{m}\right) .
$$

This completes the proof of (i).

Similarly, we can prove that

$$
\left[c^{2}, \mathcal{M}^{\prime}, p, d,\|\cdot, \cdots, \cdot\|\right]^{\theta}\left(\Delta^{\mathrm{m}}\right) \subset\left[c^{2}, \mathcal{M} \circ \mathcal{M}^{\prime}, p, d,\|\cdot, \cdots, \cdot\|\right]^{\theta}\left(\Delta^{\mathrm{m}}\right)
$$

and

$$
\left[c^{2}, \mathcal{M}^{\prime}, p, d,\|\cdot, \cdots, \cdot\|\right]_{\infty}^{\theta}\left(\Delta^{m}\right) \subset\left[c^{2}, \mathcal{M} \circ \mathcal{M}^{\prime}, p, d,\|\cdot, \cdots, \cdot\|\right]_{\infty}^{\theta}\left(\Delta^{m}\right) .
$$


Corollary 1 Let $0<\inf p_{k, l}=\mathrm{h} \leq \mathrm{p}_{\mathrm{k}, \mathrm{l}} \leq \sup \mathrm{p}_{\mathrm{k}, \mathrm{l}}=\mathrm{H}<\infty$ and $\mathcal{M}=\left(\mathrm{M}_{\mathrm{k}, \mathrm{l}}\right)$ be a sequence of Orlicz functions satisfying $\Delta_{2}$-condition, then we have

$$
\left[c^{2}, p, d,\|\cdot, \cdots, \cdot\|\right]_{0}^{\theta}\left(\Delta^{m}\right) \subset\left[c^{2}, \mathcal{M}, p, d,\|\cdot, \cdots, \cdot\|\right]_{0}^{\theta}\left(\Delta^{m}\right)
$$

and

$$
\left[c^{2}, p, d,\|\cdot, \cdots, \cdot\|\right]_{\infty}^{\theta}\left(\Delta^{m}\right) \subset\left[c^{2}, \mathcal{M}, p, d,\|\cdot, \cdots, \cdot\|\right]_{\infty}^{\theta}\left(\Delta^{m}\right) .
$$

Proof. Taking $\mathcal{M}^{\prime}(x)=x$ in the above theorem, we get the required result. $\square$

Theorem 4 Let $\mathcal{M}=\left(M_{k, l}\right)$ be a sequence of Orlicz functions. Then the following statements are equivalent:

(i) $\left[c^{2}, p, d,\|\cdot, \cdots, \cdot\|\right]_{\infty}^{\theta}\left(\Delta^{m}\right) \subset\left[c^{2}, \mathcal{M}, p, d,\|\cdot, \cdots, \cdot\|\right]_{\infty}^{\theta}\left(\Delta^{m}\right)$,

(ii) $\left[c^{2}, p, d,\|\cdot, \cdots, \cdot\|\right]_{0}^{\theta}\left(\Delta^{m}\right) \subset\left[c^{2}, \mathcal{M}, p, d,\|\cdot, \cdots, \cdot\|\right]_{\infty}^{\theta}\left(\Delta^{m}\right)$ and

(iii) $\sup _{r} \frac{1}{g_{r, s}} \sum_{k, l \in I_{r, s}}\left[M_{k, l}\left(\frac{t}{\rho}\right)\right]^{p_{k, l}}<\infty(t, \rho>0)$.

Proof. (i) $\Rightarrow$ (ii) The proof is obvious in view of the fact that

$$
\left[c^{2}, p, d,\|\cdot, \cdots, \cdot\|\right]_{0}^{\theta}\left(\Delta^{m}\right) \subset\left[c^{2}, p, d,\|\cdot, \cdots, \cdot\|\right]_{\infty}^{\theta}\left(\Delta^{m}\right) .
$$

(ii) $\Rightarrow$ (iii) Let $\left[c^{2}, p, d,\|\cdot, \cdots, \cdot\|\right]_{0}^{\theta}\left(\Delta^{m}\right) \subset\left[c^{2}, \mathcal{M}, p, d,\|\cdot, \cdots, \cdot\|\right]_{\infty}^{\theta}\left(\Delta^{m}\right)$. Suppose that (iii) does not hold. Then for some $t$, there exists $\rho>0$ such that

$$
\sup _{r, s} \frac{1}{g_{r, s}} \sum_{k, l \in I_{r, s}}\left[M_{k, l}\left(\frac{t}{\rho}\right)\right]^{p_{k, l}}=\infty
$$

and therefore we can find a subinterval $I_{r, s(j)}$ of the set of interval $I_{r, s}$ such that

$$
\frac{1}{g_{r, s(j)}} \sum_{k, l \in I_{r, s(j)}}\left[M_{k, l}\left(\frac{j^{-1}}{\rho}\right)\right]^{p_{k, l}}>j, j=1,2 .
$$

Define the sequence $x=\left(x_{k, l}\right)$ by

$$
d_{k, l} \Delta^{m} x_{k+u, l+v}=\left\{\begin{array}{ll}
j^{-1}, & k, l \in I_{r, s(j)} \\
0, & k, l \notin I_{r, s(j)}
\end{array} \quad \text { for all } u \text { and } v \in \mathbb{N} .\right.
$$

Then $x=\left(x_{k, l}\right) \in\left[c^{2}, p, d,\|\cdot, \cdots, \cdot\|\right]_{0}^{\theta}\left(\Delta^{m}\right)$ but by equation (4), 
$x=\left(x_{k, l}\right) \notin\left[c^{2}, \mathcal{M}, p, d,\|\cdot, \cdots, \cdot\|\right]_{\infty}^{\theta}\left(\Delta^{\mathrm{m}}\right)$, which contradicts (ii). Hence (iii) must hold.

(iii) $\Rightarrow$ (i) Let (iii) hold and $x=\left(x_{k, l}\right) \in\left[c^{2}, p, d,\|\cdot, \cdots, \cdot\|\right]_{\infty}^{\theta}\left(\Delta^{m}\right)$. Suppose that

$x=\left(x_{k, l}\right) \notin\left[c^{2}, \mathcal{M}, p, d,\|\cdot, \cdots, \cdot\|\right]_{\infty}^{\theta}\left(\Delta^{m}\right)$.

Then

$$
\sup _{r, s} \frac{1}{g_{r, s}} \sum_{k, l \in I_{r, s}}\left[M_{k, l}\left(\left\|\frac{d_{k, l} \Delta^{m} \chi_{k+u, l+v}}{\rho}, z_{1}, \cdots, z_{n-1}\right\|\right)\right]^{p_{k, l}}=\infty .
$$

Let $t=\left\|d_{k, l} \Delta^{m} x_{k+u, l+v}, z_{1}, \cdots, z_{n-1}\right\|$ for each $k, l$ and fixed $u, v$, then by equations (5)

$$
\sup _{r, s} \frac{1}{g_{r, s}} \sum_{k, l \in I_{r, s}}\left[M_{k, l}\left(\frac{t}{\rho}\right)\right]=\infty,
$$

which contradicts (iii). Hence (i) must hold.

Theorem 5 Let $1 \leq p_{k, l} \leq \sup _{k, l}<\infty$ and $\mathcal{M}=\left(M_{k, l}\right)$ be a sequence of Orlicz functions. Then the following statements are equivalent:

(i) $\left[c^{2}, \mathcal{M}, p, d,\|\cdot, \cdots, \cdot\|\right]_{0}^{\theta}\left(\Delta^{m}\right) \subset\left[c^{2}, p, d,\|\cdot, \cdots, \cdot\|\right]_{0}^{\theta}\left(\Delta^{m}\right)$,

(ii) $\left[\mathrm{c}^{2}, \mathcal{M}, \mathrm{p}, \mathrm{d},\|\cdot, \cdots, \cdot\|\right]_{0}^{\Theta}\left(\Delta^{\mathrm{m}}\right) \subset\left[\mathrm{c}^{2}, \mathrm{p}, \mathrm{d},\|\cdot, \cdots, \cdot\|\right]_{\infty}^{\Theta}\left(\Delta^{\mathrm{m}}\right)$ and

(iii) $\inf _{r, s} \frac{1}{g_{r, s}} \sum_{k, l \in I_{r, s}}\left[M_{k, l}\left(\frac{t}{\rho}\right)\right]^{p_{k, l}}>0(t, \rho>0)$.

Proof. (i) $\Rightarrow$ (ii) It is trivial.

(ii) $\Rightarrow$ (iii) Let (ii) hold. Suppose that (iii) does not hold. Then

$$
\inf _{r, s} \frac{1}{g_{r, s}} \sum_{k, l \in I_{r, s}}\left[M_{k, l}\left(\frac{t}{\rho}\right)\right]^{p_{k, l}}=0(t, \rho>0),
$$

so we can find a subinterval $I_{r, s(j)}$ of the set of interval $I_{r, s}$ such that

$$
\frac{1}{g_{r, s(j)}} \sum_{k, l \in I_{r, s(j)}}\left[M_{k, l}\left(\frac{j}{\rho}\right)\right]^{p_{k, l}}<j^{-1}, j=1,2, \cdots
$$

Define the sequence $x=\left(x_{k, l}\right)$ by

$$
d_{k, l} \Delta^{m} x_{k+u, l+v}=\left\{\begin{array}{ll}
j, & k, l \in I_{r, s(j)} \\
0, & k, l \notin I_{r, s(j)}
\end{array} \quad \text { for all } u \text { and } v \in \mathbb{N} .\right.
$$


Thus by equation $(6), x=\left(x_{k, l}\right) \in\left[c^{2}, \mathcal{M}, p, d,\|\cdot, \cdots, \cdot\|\right]_{0}^{\theta}\left(\Delta^{m}\right)$, but $x=\left(x_{k, l}\right) \notin$ $\left[c^{2}, p, d,\|\cdot, \cdots, \cdot\|\right]_{\infty}^{\theta}\left(\Delta^{m}\right)$, which contradicts (ii). Hence (iii) must hold.

(iii) $\Rightarrow$ (i) Let (iii) hold and suppose that $x=\left(x_{k, l}\right) \in\left[c^{2}, \mathcal{M}, p, d,\|\cdot, \cdots, \cdot\|\right]_{0}^{\theta}$ $\left(\Delta^{\mathrm{m}}\right)$, i.e,

$$
\lim _{r, s \rightarrow \infty} \frac{1}{g_{r, s}} \sum_{k, l \in I_{r, s}}\left[M_{k, l}\left(\left\|\frac{d_{k, l} \Delta^{m} \chi_{k+u, l+v}}{\rho}, z_{1}, \cdots, z_{n-1}\right\|\right)\right]^{p_{k, l}}=0,
$$

uniformly in $u$ and $v$, for some $\rho>0$.

Again, suppose that $x=\left(x_{k, l}\right) \notin\left[c^{2}, p, d, \| \cdot, \cdots, \cdot\right]_{0}^{\theta}\left(\Delta^{m}\right)$. Then, for some number $\epsilon>0$ and a subinterval $\mathrm{I}_{r, s(j)}$ of the set of interval $\mathrm{I}_{r, s}$, we have $\left\|\mathrm{d}_{\mathrm{k}, \mathrm{l}} \Delta^{\mathrm{m}} \mathrm{x}_{\mathrm{k}+\mathrm{u}, \mathrm{l}+v}, z_{1}, \cdots, z_{n-1}\right\| \geq \epsilon$ for all $\mathrm{k} \in \mathbb{N}$ and some $u \geq \mathfrak{u}_{0}, v \geq v_{0}$. Then, from the properties of the Orlicz function, we can write

$$
M_{k, l}\left(\left\|\frac{d_{k, l} \Delta^{m} \chi_{k+u, l+v}}{\rho}, z_{1}, \cdots, z_{n-1}\right\|\right)^{p_{k, l}} \geq M_{k, l}\left(\frac{\epsilon}{\rho}\right)^{p_{k, l}}
$$

and consequently by (7)

$$
\lim _{r, s \rightarrow \infty} \frac{1}{g_{r, s}} \sum_{k, l \in I_{r, s}}\left[M_{k, l}\left(\frac{\epsilon}{\rho}\right)\right]^{p_{k, l}}=0,
$$

which contradicts (iii). Hence (i) must hold.

Proposition 2 Let $0<p_{k, l} \leq q_{k, l}$ for all $k, l \in \mathbb{N}$ and $\left(\frac{q_{k, l}}{p_{k, l}}\right)$ be bounded. Then, $\left[c^{2}, \mathcal{M}, q, d,\|\cdot, \cdots, \cdot\|\right]^{\theta}\left(\Delta^{\mathrm{m}}\right) \subset\left[\mathrm{c}^{2}, \mathcal{M}, \mathrm{p}, \mathrm{d},\|\cdot, \cdots, \cdot\|\right]^{\theta}\left(\Delta^{\mathrm{m}}\right)$.

Proof. Let $x \in\left[c^{2}, \mathcal{M}, q, d,\|\cdot, \cdots, \cdot\|\right]^{\theta}\left(\Delta^{m}\right)$. Write

$$
t_{k, l}=\left[M_{k, l}\left(\left\|\frac{d_{k, l} \Delta^{m} x_{k+u, l+v}-L}{\rho}, z_{1}, \cdots, z_{n-1}\right\|\right)\right]^{q_{k, l}}
$$

and $\mu_{k, l}=\frac{p_{k, l}}{q_{k, l}}$ for all $k, l \in \mathbb{N}$. Then $0<\mu_{k, l} \leq 1$ for $k, l \in \mathbb{N}$. Take $0<\mu<$ $\mu_{k, l}$ for $k, l \in \mathbb{N}$. Define the sequences $\left(a_{k, l}\right)$ and $\left(b_{k, l}\right)$ as follows: For $t_{k, l} \geq 1$, let $a_{k, l}=t_{k, l}$ and $b_{k, l}=0$ and for $t_{k, l}<1$, let $a_{k, l}=0$ and $b_{k, l}=t_{k, l}$. Then clearly for all $k, l \in \mathbb{N}$, we have

$$
t_{k, l}=a_{k, l}+b_{k, l}, \quad t_{k, l}^{\mu_{k, l}}=a_{k, l}^{\mu_{k, l}}+b_{k, l}^{\mu_{k, l}} .
$$


Now it follows that $a_{k, l}^{\mu_{k, l}} \leq a_{k, l} \leq t_{k, l}$ and $b_{k, l}^{\mu_{k, l}} \leq b_{k, l}^{\mu}$. Therefore,

$$
\begin{aligned}
\frac{1}{g_{r, s}} \sum_{k, l \in I_{r, s}} t_{k, l}^{\mu_{k, l}} & =\frac{1}{g_{r, s}} \sum_{k, l \in I_{r, s}}\left(a_{k, l}^{\mu_{k, l}}+b_{k, l}^{\mu_{k, l}}\right) \\
& \leq \frac{1}{g_{r, s}} \sum_{k, l \in I_{r, s}} t_{k, l}+\frac{1}{g_{r, s}} \sum_{k, l \in I_{r, s}} b_{k, l}^{\mu} .
\end{aligned}
$$

Now for each $k$ and $l$,

$$
\begin{aligned}
\frac{1}{g_{r, s}} \sum_{k, l \in I_{r, s}} b_{k, l}^{\mu} & =\sum_{k, l \in I_{r, s}}\left(\frac{1}{g_{r, s}} b_{k, l}\right)^{\mu}\left(\frac{1}{g_{r, s}}\right)^{1-\mu} \\
& \leq\left(\sum_{k, l \in I_{r, s}}\left[\left(\frac{1}{g_{r, s}} b_{k, l}\right)^{\mu}\right]^{\frac{1}{\mu}}\right)^{\mu}\left(\sum_{k, l \in I_{r, s}}\left[\left(\frac{1}{g_{r, s}}\right)^{1-\mu}\right]^{\frac{1}{1-\mu}}\right)^{1-\mu} \\
& =\left(\frac{1}{g_{r, s}} \sum_{k, l \in I_{r, s}} b_{k, l}\right)^{\mu}
\end{aligned}
$$

and so

$$
\frac{1}{g_{r, s}} \sum_{k, l \in I_{r, s}} t_{k, l}^{\mu_{k, l}} \leq \frac{1}{g_{r, s}} \sum_{k, l \in I_{r, s}} t_{k, l}+\left(\frac{1}{g_{r, s}} \sum_{k, l \in I_{r, s}} b_{k, l}\right)^{\mu} .
$$

Hence $x=\left(x_{k, l}\right) \in\left[c^{2}, \mathcal{M}, p, d,\|\cdot, \cdots, \cdot\|\right]^{\theta}\left(\Delta^{m}\right)$.

Theorem 6 (a) If $0<\inf p_{k, l} \leq p_{k, l} \leq 1$ for all $k, l \in \mathbb{N}$, then

$$
\left[c^{2}, \mathcal{M}, p, d,\|\cdot, \cdots, \cdot\|\right]^{\theta}\left(\Delta^{\mathrm{m}}\right) \subset\left[\mathrm{c}^{2}, \mathcal{M}, \mathrm{d},\|\cdot, \cdots, \cdot\|\right]^{\theta}\left(\Delta^{\mathrm{m}}\right) .
$$

(b) If $1 \leq p_{k, l} \leq \sup p_{k, l}<\infty$, for all $k, l \in \mathbb{N}$. Then

$$
\left[c^{2}, \mathcal{M}, \mathrm{d},\|\cdot, \cdots, \cdot\|\right]^{\theta}\left(\Delta^{\mathrm{m}}\right) \subset\left[\mathrm{c}^{2}, \mathcal{M}, \mathrm{p}, \mathrm{d},\|\cdot, \cdots, \cdot\|\right]^{\theta}\left(\Delta^{\mathrm{m}}\right) .
$$

Proof. (a) Let $x=\left(x_{k, l}\right) \in\left[c^{2}, \mathcal{M}, p, d,\|\cdot, \cdots, \cdot\|\right]^{\theta}\left(\Delta^{m}\right)$, then

$$
\lim _{r, s \rightarrow \infty} \frac{1}{g_{r, s}} \sum_{k, l \in I_{r, s}}\left[M_{k, l}\left(\left\|\frac{d_{k, l} \Delta^{m} \chi_{k+u, l+v}-L}{\rho}, z_{1}, \cdots, z_{n-1}\right\|\right)\right]^{p_{k, l}}=0 .
$$


Since $0<\inf p_{k, l} \leq p_{k, l} \leq 1$. This implies that

$$
\begin{aligned}
\lim _{r, s \rightarrow \infty} \frac{1}{g_{r, s}} & \sum_{k, l \in I_{r, s}}\left[M_{k, l}\left(\left\|\frac{d_{k, l} \Delta_{x_{k+u}, l+v}^{m}-L}{\rho}, z_{1}, \cdots, z_{n-1}\right\|\right)\right] \\
\leq & \lim _{r, s \rightarrow \infty} \frac{1}{g_{r, s}} \sum_{k, l \in I_{r, s}}\left[M_{k, l}\left(\left\|\frac{d_{k, l} \Delta^{m} x_{k+u, l+v}-L}{\rho}, z_{1}, \cdots, z_{n-1}\right\|\right)\right]^{p_{k, l}} .
\end{aligned}
$$

Therefore, $\lim _{r, s \rightarrow \infty} \frac{1}{g_{r, s}} \sum_{k, l \in I_{r, s}}\left[M_{k, l}\left(\left\|\frac{d_{k, l} \Delta^{m} x_{k+u, l+v}-L}{\rho}, z_{1}, \cdots, z_{n-1}\right\|\right)\right]=0$.

This shows that $x=\left(x_{k, l}\right) \in\left[c^{2}, \mathcal{M}, d,\|\cdot, \cdots, \cdot\|\right]^{\theta}\left(\Delta^{m}\right)$.

Therefore,

$$
\left[c^{2}, \mathcal{M}, p, d,\|\cdot, \cdots, \cdot\|\right]^{\theta}\left(\Delta^{m}\right) \subset\left[c^{2}, \mathcal{M}, d,\|\cdot, \cdots, \cdot\|\right]^{\theta}\left(\Delta^{m}\right) .
$$

This completes the proof.

(b) Let $p_{k, l} \geq 1$ for each $k, l$ and $\sup p_{k, l}<\infty$. Let $x=\left(x_{k, l}\right) \in\left[c^{2}, \mathcal{M}, d\right.$, $\|\cdot, \cdots, \cdot\|]^{\theta}\left(\Delta^{\mathrm{m}}\right)$. Then for each $\epsilon>0$ there exists a positive integer $\mathrm{N}$ such that

$$
\lim _{r, s \rightarrow \infty} \frac{1}{g_{r, s}} \sum_{k, l \in I_{r, s}}\left[M_{k, l}\left(\left\|\frac{d_{k, l} \Delta^{m} x_{k+u, l+v}-L}{\rho}, z_{1}, \cdots, z_{n-1}\right\|\right)\right]=0<1 .
$$

Since $1 \leq p_{k, l} \leq \sup p_{k, l}<\infty$, we have

$$
\begin{aligned}
\lim _{r, s \rightarrow \infty} \frac{1}{g_{r, s}} & \sum_{k, l \in I_{r}, s}\left[M_{k, l}\left(\left\|\frac{d_{k, l} \Delta^{m} x_{k+u, l+v}-L}{\rho}, z_{1}, \cdots, z_{n-1}\right\|\right)\right]^{p_{k, l}} \\
\leq & \lim _{r, s \rightarrow \infty} \frac{1}{g_{r, s}} \sum_{k, l \in I_{r, s}}\left[M_{k, l}\left(\| \frac{d_{k, l} \Delta^{m} x_{k+u, l+v}-L}{\rho}, z_{1}, \cdots, z_{n-1} \mid\right)\right] \\
& =0<1 .
\end{aligned}
$$

Therefore $x=\left(x_{k, l}\right) \in\left[c^{2}, \mathcal{M}, p, d,\|\cdot, \cdots, \cdot\|\right]^{\theta}\left(\Delta^{m}\right)$.

\section{Acknowledgement}

The authors are thankful to the referee for his valuable comments which improve the presentation of the paper. 


\section{References}

[1] B. Altay, F. Basar, Some new spaces of double sequencs, J. Math. Anal. Appl., 309 (2005), 70-90.

[2] M. Basarir, O. Sonalcan, On some double sequence spaces, J. Indian Acad. Math., 21 (1999), 193-200.

[3] F. Basar, Y. Sever, The space $\mathcal{L}_{\mathrm{p}}$ of double sequences, Math. J. Okayama Univ., 51 (2009), 149-157.

[4] T. J. Bromwich, An introduction to the theory of infinite series, Macmillan and co. Ltd., New York (1965).

[5] M. Et, R. Colak, On generalized difference sequence spaces, Soochow J. Math. 21 (1995), 377-386.

[6] A. R. Freedman, J. J.Sember, M. Raphael, Some Cesaro-type summability spaces, Proc. London Math. Soc., 37 (1978), 508-520.

[7] S. Gahler, Linear 2-normietre Rume, Math. Nachr., 28 (1965), 1-43.

[8] H. Gunawan, On n-Inner Product, n-Norms, and the Cauchy-Schwartz Inequality, Scientiae Mathematicae Japonicae, 5 (2001), 47-54.

[9] H. Gunawan, The space of p-summable sequence and its natural n-norm, Bull. Aust. Math. Soc., 64 (2001), 137-147.

[10] H. Gunawan, M. Mashadi, On n-normed spaces, Int. J. Math. Math. Sci., 27 (2001), 631-639.

[11] G. H. Hardy, On the convergence of certain multiple series, Proc. Camb. Phil. Soc., 19 (1917), 86-95.

[12] H. Kizmaz, On certain sequence spaces, Canad. Math-Bull., 24 (1981), $169-176$.

[13] G. G. Lorentz, A contribution to the theory of divergent sequences, Acta Mathematica, 80 (1948), 167-190.

[14] J. Lindenstrauss, L. Tzafriri, On Orlicz sequence spaces, Israel J. Math., 10 (1971), 345-355. 
[15] I. J. Maddox, Spaces of strongly summable sequences, Quart. J. Math., 18 (1967), 345-355.

[16] I. J. Maddox, A new type of convergence, Math. Proc. Camb. Phil. Soc., 83 (1978), 61-64.

[17] L. Maligranda, Orlicz spaces and interpolation, Seminars in Mathematics 5, Polish Academy of Science, 1989.

[18] F. Moricz, Extension of the spaces $c$ and $c_{0}$ from single to double sequences, Acta Math. Hungarica, 57 (1991), 129-136.

[19] F. Moricz, B. E. Rhoades, Almost convergence of double sequences and strong reqularity of summability matrices, Math. Proc. Camb. Phil. Soc., 104 (1988), 283-294.

[20] A. Misiak, n-inner product spaces, Math. Nachr., 140 (1989), 299-319.

[21] M. Mursaleen, Almost strongly regular matrices and a core theorem for double sequences, J. Math. Anal. Appl., 293(2),(2004), 523-531.

[22] M. Mursaleen, M. A. Khan, Qamaruddin, Difference sequence spaces defined by Orlicz functions, Demanstratio Math. Vol. XXXII(1999), 145150 .

[23] M. Mursaleen, O. H. H. Edely, Statistical convergence of double sequences, J. Math. Anal. Appl., 288 (1),(2003), 223-231.

[24] M. Mursaleen, O. H. H. Edely, Almost convergence and a core theorem for double sequences, J. Math. Anal. Appl., 293 (2),(2004), 532-540.

[25] J. Musielak, Orlicz spaces and modular spaces, Lecture Notes in Mathematics, 1034 (1983).

[26] H. Nakano, Modular sequence spaces, Proc. Japan Acad., 27 (1951), 508512.

[27] A. Pringsheim, Zur Theori der zweifach unendlichen Zahlenfolgen, Math. Ann. 53(1900), 289-321.

[28] K. Raj, A. K. Sharma, S. K. Sharma, A Sequence space defined by Musielak-Orlicz functions, Int. J. Pure and Appl. Math., 67 (2011), 475484. 
[29] K. Raj, S. K. Sharma, A. K. Sharma, Some difference sequence spaces in n-normed spaces defined by Musielak-Orlicz function, Armen. J. Math., 3 (2010), 127-141.

[30] K. Raj, S. K. Sharma, Some sequence spaces in 2-normed spaces defined by Musielak-Orlicz function, Acta Univ. Sapientiae Mathematica, 3 (2011), 97-109.

[31] D. Rath, B. C. Tripathy, Matrix maps on sequence spaces associated with sets of integers, Indian J. Pure Appl. Math., 27 (1996), 197-206.

[32] E. Savas, Some new double sequence spaces defined by Orlicz function in n-normed space, J. Inequal. Appl., Vol. 2011, ID 592840, 1-9.

[33] S. Simons, The sequence spaces $l\left(\mathrm{p}_{v}\right)$ and $\mathrm{m}\left(\mathrm{p}_{v}\right)$, Proc. Japan Acad., 27 (1951), 508-512.

[34] I. J. Schoenberg, The integrability of certain functions and related summability methods, Amer. Math. Monthly, 66 (1959), 3621-4375.

[35] B. C. Tripathy, Generalized difference paranormed statistically convergent sequences defined by Orlicz function in a locally convex spaces, Soochow J. Math., 30 (2004), 431-446.

[36] B. C. Tripathy, Statistically convergent double sequences, Tamkang J. Math., 34 (2003), 231-237.

[37] A. Wilansky, Summability through Functional Analysis, North- Holland Math. Stud. 85(1984).

[38] M. Zeltser, Investigation of double sequence spaces by Soft and Hard Analytical Methods, Dissertationes Mathematicae Universitatis Tartuensis 25, Tartu University Press, Univ. of Tartu, Faculty of Mathematics and Computer Science, Tartu (2001). 